\title{
Prácticas de Gestión del Conocimiento en una Institución Pública de Investigación. El Caso del Centro Tecnológico del Ejército en Brasil (CTEx)
}

\author{
Ralfh V. Ansuattigui, Antonio J. Caulliraux-Pithon y José L. Fernandes \\ Centro Federal de Educación Tecnológica Celso Suckow da Fonseca, Dirección de Investigación y \\ Posgrado, Av. Maracanã, 229 - Bloco E - 5 ${ }^{\circ}$ andar, 20271-110 RJ-Brasil, (e-mail: caulliraux@gmail.com)
}

Recibido Ene. 29, 2013; Aceptado Mar. 21, 2013; Versión final recibida May. 08, 2013

\begin{abstract}
Resumen
Se presenta un estudio sobre el uso de prácticas de gestión, que pueden ser calificadas como prácticas de gestión del conocimiento, en el Centro Tecnológico del Ejército CTEx, una institución pública de investigación en Brasil. Para ello, se aplicó un cuestionario dividido en tres partes: la primera investiga los antecedentes académicos de los miembros de la institución en especial si tenía algún tipo de formación en gestión; la segunda formada por un conjunto de veinte preguntas referidas a las diferentes prácticas de gestión existentes en la institución; y la tercera en que se plantea una pregunta abierta sobre una de estas prácticas de gestión y que ellos consideran de especial importancia. Los resultados mostraron que el CTEx no posee un plan estructurado de gestión y además no aplica en forma adecuada la mayoría de las mencionadas prácticas de gestión.
\end{abstract}

Palabras clave: prácticas de gestión, gestión del conocimiento, diagnóstico de la gestión, gestión pública

\section{Knowledge Management Practices in a Public Research Institute: The Case of Technological Center of the Army in Brazil (CTEx)}

\begin{abstract}
This paper presents a study on the use of management practices, which can be classified as knowledge management practices, at the Technological Center of the Army, CTEx, a public research institution in Brazil. For this, a questionnaire divided into three parts was applied: the first part investigates the academic background of the members of the institution, especially inquiring about previous training in management they might have; the second part consisted of a set of twenty questions related to the different management practices existing in the institution; and the third part that included an open question about one of the existing practices that they considered that deserve special attention. The results showed that the CTEx not only does not have a structured management plan but also it does not properly apply most of the existing management practices.
\end{abstract}

Keywords: management practices, knowledge management, management diagnosis, public management 


\section{INTRODUCCIÓN}

A pesar de la "poca edad" de los estudios relativos a la Gestión del Conocimiento, al examinar de forma rápida y superficial la historia del desarrollo de la humanidad, ella nos revela que el conocimiento siempre ofreció ventajas a aquellos que supieron aplicarlo, como puede ser visto en las palabras de Alfred Marshall al argumentar que "El valor económico de un único genio industrial es suficiente para cubrir los gastos de educación de toda una ciudad, pues una única idea, como, por ejemplo, el gran invento de Bessemer, aumenta tanto la capacidad productiva de Inglaterra como el trabajo de cien mil hombres." (Tigre, 2006).

Toffler (1980) afirmó hace tres décadas que en la sociedad post-revolución industrial, por él llamada de Sociedad del Conocimiento, los diversos segmentos de la economía serían liderados por las organizaciones innovadoras, en las cuales el conocimiento suplantaría en valor e importancia los factores productivos tradicionales. Esta afirmación es ratificada por (Alee y Taug, 2006) que preconizan que el capital financiero como recurso primario de ventaja competitiva fue sustituido por el conocimiento e información con el auxilio de la conectividad digital y del creciente acceso a la información.

Considerando, entonces, la importancia de planificar previamente la implantación de un programa de Gestión del Conocimiento tratando de asegurar que esta iniciativa pueda agregar valor a las actividades de una organización, seria importante ejecutar un diagnóstico del estado de las actividades y prácticas relacionadas a la Gestión del Conocimiento como punto de partida. Como puede ser visto en Coombs et. al. (1998); Henczel, Liebowitz et al. (2000); Hylton (2002); Burnett et. al. (2004); Perez-Soltero (2009) y Cheung et al. (2007), el diagnóstico de los factores críticos pasibles de impactar en la adopción de la Gestión del Conocimiento, como también de las prácticas y actividades relacionadas con esta iniciativa, debe ser objeto de cuidadoso planeamiento y ejecución a través de una Auditoria del Conocimiento, práctica considerada fundamental y que debe, obligatoriamente, figurar como primera etapa del planeamiento de una iniciativas de Gestión del Conocimiento.

El objetivo general de este artículo es identificar la existencia de Prácticas de Gestión en una Institución Pública de Investigación en Ciencia, Tecnología e Innovación, específicamente, el Centro Tecnológico del Ejército (CTEX), las cuales sean compatibles con las Prácticas de la Gestión del Conocimiento identificadas en la literatura sobre el asunto.

\section{GESTIÓN DEL CONOCIMIENTO EN INVESTIGACIÓN Y DESARROLLO}

Amidon (1996), en una investigación sobre la evolución de los Sistemas de Gestión de Investigación y Desarrollo, mostró que fuerzas económicas, comportamentales y tecnológicas, conducirían al hombre a una nueva era en la cual el foco sería el conocimiento. $Y$, también, bajo tales condiciones, la autora preconizó que los sistemas de gestión de investigación y desarrollo deberían evolucionar y contemplar cinco grandes transformaciones que irían a influenciar definitivamente el mercado mundial:

a) El conocimiento como fuente de riqueza se volvería más importante que los factores tradicionales (tierra, capital y trabajo) y, de esta forma, el capital intelectual debe ser administrado como un activo de la organización.

b) Los diseños organizacionales tradicionalmente jerárquicos, oriundos de la era industrial, darían lugar a formas de organización en red, lo suficientemente flexibles para dar cabida a la capacidad intelectual intrínseca de las organizaciones.

c) El papel de la educación se volvería prominente para todos los tipos de organizaciones privadas o públicas y la orientación pasiva entrenamiento/desarrollo daría lugar a una perspectiva activa con foco en el aprendizaje en tiempo real.

d) Para mantener la rentabilidad, las organizaciones deberían seguir la ola de la globalización y, de esta forma, sus estrategias pasarían a tener un foco internacional;

e) Las estrategias de las organizaciones deberían privilegiar la colaboración en detrimento de la competición, para crear escenarios del tipo vencedor/vencedor en lugar del tipo vencedor/perdedor.

En las dos últimas décadas, otros autores efectuaron investigaciones buscando establecer relaciones entre la Gestión del Conocimiento y la Investigación y Desarrollo bajo una perspectiva evolutiva de los sistemas de gestión de investigación y desarrollo y sus respectivas necesidades con referencia a la Gestión del Conocimiento. Park y Kim (2005), consolidando de forma consistente el resultado de tales investigaciones, afirmaron que la relación existente entre la Gestión del Conocimiento y la Investigación y Desarrollo es intrínsecamente próximo, ya que los procesos de investigación y desarrollo pueden ser vistos primariamente como procesos de Gestión del Conocimiento. Tales procesos, en la visión de estos autores, son 
responsables por transformar la información en progresos tecnológicos y las demandas de mercado en el conocimiento necesario para nuevos conceptos de productos y proyectos de procesos. La organización de Investigación y Desarrollo, por ser la principal fuente de generación y diseminación de conocimiento, desempeña el papel de actor primario en el proceso de la Gestión del Conocimiento.

Park y Kim (2005) observando que, tal cual el proceso de innovación, la Gestión del Conocimiento y la Investigación y Desarrollo transitaban un camino evolutivo, en el cual los estándares de los principios y prácticas de gestión estaban en franco cambio, propusieron una clasificación evolutiva de los sistemas de gestión de Investigación y Desarrollo abarcando cuatro generaciones. La Tabla 1 presenta las principales características de cada una de esas cuatro generaciones de sistemas de gestión de Investigación y Desarrollo (I+D).

Tabla 1. Características de los Sistemas de Gestión de Investigación y Desarrollo

\begin{tabular}{|l|l|l|l|}
\hline \multicolumn{1}{|c|}{$1^{\text {a }}$ Generación } & \multicolumn{1}{|c|}{$2^{\text {a Generación }}$} & \multicolumn{1}{c|}{$3^{\text {a Generación }}$} & \multicolumn{1}{c|}{ 4 $^{\text {a Generación }}$} \\
\hline $\begin{array}{l}\text { I+D orientada al } \\
\text { Investigador }\end{array}$ & $\begin{array}{l}\text { I+D orientada al } \\
\text { Administrador }\end{array}$ & I+D orientada a la Empresa & I+D orientada al Cliente \\
\hline $\begin{array}{l}\text { Modelo de innovación } \\
\text { Linear }\end{array}$ & $\begin{array}{l}\text { Modelo de innovación } \\
\text { parcialmente no linear }\end{array}$ & $\begin{array}{l}\text { Modelo de innovación } \\
\text { Encadenada }\end{array}$ & $\begin{array}{l}\text { Modelo de innovación de } \\
\text { sistema y red }\end{array}$ \\
\hline $\begin{array}{l}\text { Investigación científica } \\
\text { y básica }\end{array}$ & Investigación aplicada & $\begin{array}{l}\text { Portafolio equilibrado de } \\
\text { tipos de I+D }\end{array}$ & $\begin{array}{l}\text { Gestión del ciclo de vida } \\
\text { de I+D }\end{array}$ \\
\hline $\begin{array}{l}\text { Planeamiento } \\
\text { impulsado por la oferta } \\
\text { de tecnología }\end{array}$ & $\begin{array}{l}\text { Planeamiento impulsado } \\
\text { por la oferta de } \\
\text { tecnología y demanda } \\
\text { de mercado }\end{array}$ & $\begin{array}{l}\text { Planeamiento impulsado } \\
\text { por la demanda de mercado } \\
\text { y relación con los } \\
\text { proveedores }\end{array}$ & $\begin{array}{l}\text { Énfasis en proyectos } \\
\text { dominantes }\end{array}$ \\
\hline $\begin{array}{l}\text { Organización } \\
\text { jerárquica de I+D }\end{array}$ & $\begin{array}{l}\text { Organización matricial } \\
\text { de I+D }\end{array}$ & $\begin{array}{l}\text { Organización distribuida de } \\
\text { I+D }\end{array}$ & $\begin{array}{l}\text { Liderazgo de investiga- } \\
\text { dores innovadores }\end{array}$ \\
\hline $\begin{array}{l}\text { Virtualmente ninguna } \\
\text { auditoria }\end{array}$ & $\begin{array}{l}\text { Guión tecnológico para el } \\
\text { planeamiento de I\&D y } \\
\text { Gestión de la Propiedad } \\
\text { Intelectual }\end{array}$ & $\begin{array}{l}\text { Organización global y } \\
\text { colaborativa }\end{array}$ \\
\hline Mínima documentación proyectos & $\begin{array}{l}\text { Procesamiento basado } \\
\text { en datos }\end{array}$ & $\begin{array}{l}\text { Procesamiento basado en } \\
\text { la información }\end{array}$ & $\begin{array}{l}\text { Procesamiento basado en } \\
\text { el conocimiento y Gestión } \\
\text { online }\end{array}$ \\
\hline
\end{tabular}

\section{El concepto del conocimiento}

Davenport y Prusak (2003) definen que: "El Conocimiento es una mezcla fluida de experiencia condensada, valores, información contextual e insight experimentado, la cual proporciona una estructura para la evaluación e incorporación de nuevas experiencias e informaciones. Se origina y es aplicado en la mente de los conocedores. En las organizaciones, generalmente esta embutido no sólo en documentos o repositorios, sino que también en rutinas, procesos, prácticas y normas organizacionales".

Tabla 2. Definiciones de datos, información y conocimiento

\begin{tabular}{|c|c|c|c|}
\hline Autor & Datos & Información & Conocimiento \\
\hline Wiig, 1999 & - & $\begin{array}{l}\text { Hechos organizados para describir } \\
\text { una situación o condición }\end{array}$ & $\begin{array}{l}\text { Verdades, creencias, perspectivas, } \\
\text { juzgamientos, know-how y } \\
\text { metodologías. }\end{array}$ \\
\hline $\begin{array}{l}\text { Nonaka \& } \\
\text { Takeuchi, } 1995\end{array}$ & ( & Un flujo de mensajes significativos & $\begin{array}{l}\text { Compromisos y creencias creados a } \\
\text { partir de estos mensajes }\end{array}$ \\
\hline $\begin{array}{l}\text { Spek \& } \\
\text { Spijkervet, } 1997\end{array}$ & $\begin{array}{l}\text { Símbolos aún no } \\
\text { interpretados }\end{array}$ & Datos con significado & Habilidad para atribuir significado \\
\hline $\begin{array}{l}\text { Davenport, } \\
1997\end{array}$ & Simples observaciones & Datos con relevancia y objetivo & $\begin{array}{l}\text { Informaciones valiosas originadas en } \\
\text { la mente humana }\end{array}$ \\
\hline $\begin{array}{l}\text { Davenport \& } \\
\text { Prusak, } 1998\end{array}$ & $\begin{array}{l}\text { Un conjunto de hechos } \\
\text { discretos }\end{array}$ & $\begin{array}{l}\text { Un mensaje destinado a cambiar } \\
\text { la percepción del receptor }\end{array}$ & $\begin{array}{l}\text { Experiencia, valores, discernimiento } \\
\text { e información contextual. }\end{array}$ \\
\hline $\begin{array}{l}\text { Quigley \& } \\
\text { Debons, } 1999\end{array}$ & $\begin{array}{l}\text { Texto que no responde } \\
\text { cuestiones sobre un } \\
\text { problema particular }\end{array}$ & $\begin{array}{l}\text { Texto que responde las preguntas: } \\
\text { quién ,cuándo ,qué y donde }\end{array}$ & $\begin{array}{l}\text { Texto que responde las preguntas: } \\
\text { por qué y cómo }\end{array}$ \\
\hline $\begin{array}{l}\text { Choo et. al., } \\
2000\end{array}$ & Hechos y mensajes & Datos conteniendo significado & Creencias verdaderas, justificadas. \\
\hline
\end{tabular}


Stenmark (2001) destaca que la literatura de la Gestión del Conocimiento es rica en diferentes y, más o menos, explícitos intentos de definición del conocimiento con relación a datos e información como presentados resumidamente en la tabla 2.

\section{Gestión del Conocimiento}

Sveiby (2001) preconiza que la Gestión del Conocimiento no evolucionó a partir de un conjunto de metodologías formales y que fue investigada en diferentes partes del mundo bajo, por lo menos tres perspectivas igualmente distintas. Se destacan una perspectiva originada en los Estados Unidos, influenciada por la Tecnología de la Información y con foco principal en la Información; otra perspectiva originada en Japón, con foco en la creación del Conocimiento e Innovación; y una perspectiva oriunda de Suecia y con énfasis en estrategia y medición. El autor argumenta que la perspectiva americana de la Gestión del Conocimiento contrasta con las perspectivas japonesa y sueca.

El objetivo global de la Gestión del Conocimiento en una organización es la maximización de la eficacia de las actividades a él relacionadas, como también de los retornos de los activos de conocimiento y su constante renovación. Bajo una perspectiva gerencial, la Gestión del Conocimiento sistemática abarca cuatro áreas de énfasis, o sea, monitoreo y facilitación de las actividades relacionadas con el conocimiento; creación y mantenimiento de la infraestructura del conocimiento; renovación, organización y transformación de activos de conocimiento; aplicación de los activos de conocimiento para la realización de su valor. (Wiig apud Ansuattigui e Pithon, 2011). Gestión del Conocimiento no es una disciplina única, al contrario, es un campo integrado que se basa en varias disciplinas que permiten la búsqueda de iniciativas en los diversos dominios y niveles de jerarquía organizacional (operativo, táctico y estratégico).

En la propuesta presentada en este trabajo, la Tabla 3 presenta las Prácticas de Gestión del Conocimiento en la Administración Pública Directa (Gobierno de Brasil), en el que el Comando del Ejército. Por lo tanto, podemos concluir que los estudios presentados por los autores Batista et al. (2005), Camões (2010) y Nevis (2011), no ha habido una Práctica de la Gestión del Conocimiento, lo que confirma la investigación Ansuattigui (2012).

Tabla 3. Comparación entre los autores sobre las Prácticas de Gestión del Conocimiento

\begin{tabular}{|c|c|c|}
\hline \multicolumn{3}{|c|}{ Prácticas de Gestión del Conocimiento } \\
\hline Autores & Metodología & Resultados \\
\hline $\begin{array}{l}\text { Batista et } \\
\text { al.(2005) }\end{array}$ & $\begin{array}{l}\text { Evaluada la situación de las Prácticas de Gestión } \\
\text { del Conocimiento en el Comando del Ejército }\end{array}$ & $\begin{array}{l}\text { Los resultados mostraron muy incipiente como } \\
\text { resultado de iniciativas aisladas y esfuerzos } \\
\text { dispersos. }\end{array}$ \\
\hline $\begin{array}{l}\text { Camões } \\
(2010)\end{array}$ & $\begin{array}{l}\text { Propuesta de un método para la aplicación de la } \\
\text { herramienta OKA (Organizational Knowledge } \\
\text { Management) del Banco Mundial, en el } 7^{\circ} \text { Centro } \\
\text { Integrado de Telemática, una institución } \\
\text { dependiente del Ministerio de Ciencia y Tecnología } \\
\text { del Ejército brasileño }\end{array}$ & $\begin{array}{l}\text { Como debilidades destacan la adaptación de } \\
\text { la gestión del conocimiento con incentivos } \\
\text { estratégicos y culturales de la organización. } \\
\text { Conocimiento y aprendizaje de la } \\
\text { infraestructura y el acceso al conocimiento } \\
\text { son los puntos fuertes. }\end{array}$ \\
\hline $\begin{array}{l}\text { Neves } \\
(2011)\end{array}$ & $\begin{array}{l}\text { La metodología utilizada OKA para diagnosticar } \\
\text { Gestión del Conocimiento en Centro Tecnológico } \\
\text { del Ejército }\end{array}$ & $\begin{array}{l}\text { Bajo la comprensión de los servidores de la } \\
\text { institución en cuanto el concepto y los } \\
\text { beneficios de la aplicación de la metodología y } \\
\text { la falta de planificación. }\end{array}$ \\
\hline $\begin{array}{l}\text { Ansuattigui } \\
\text { (2012) }\end{array}$ & $\begin{array}{l}\text { Trató de identificar la práctica de la gestión del } \\
\text { conocimiento aplicado actualmente en CTEx }\end{array}$ & $\begin{array}{l}\text { No se observó durante el estudio de la } \\
\text { existencia de una planificación formal en } \\
\text { relación con la gestión del conocimiento. }\end{array}$ \\
\hline
\end{tabular}

\section{RECOLECCIÓN DE DATOS}

Considerada la facilidad de envío y la flexibilidad ofrecida al participante con relación a la elección del adecuado momento para responderlo, se optó por la aplicación del cuestionario como instrumento de recolección de datos de esta investigación (Ansuattigui, 2012). Para diagnosticar el estado de la Gestión del Conocimiento en CTEx, el Banco Mundial, a través del World Bank Institute - WBI, desarrolló el método Organizational Knowledge Assessment - OKA. Este método permite la recogida de datos sobre diversos aspectos de la Gestión del Conocimiento en una organización y presenta los datos en un diagrama de radar fácil visualización e interpretación. De acuerdo con el método OKA, la Gestión del Conocimiento depende fundamentalmente los tres elementos que componen una organización: Personas, Procesos y Sistemas. 
El cuestionario de recolección de datos fue estructurado de la siguiente forma:

a) En la primera parte del cuestionario, más allá de la formación académica y del departamento donde actúa en la institución, a la persona se le pregunta si ya recibió algún tipo de entrenamiento formal en Gestión del Conocimiento. A continuación se presenta la primera pregunta abierta del cuestionario, para capturar el entendimiento de cada entrevistado sobre lo que es la gestión del conocimiento.

b) La segunda parte del cuestionario cuenta con un total de veinte cuestiones, cada una de ellas refiriéndose a una diferente práctica de gestión del conocimiento. Más allá del nombre por el cual la práctica es conocida en el medio organizacional, hay una resumida descripción que busca pasar una idea general sobre el concepto de la misma. Aquí se destaca que la idea central de este trabajo es la identificación de una o más prácticas de gestión aplicadas al trabajo diario de la institución y que puedan ser clasificadas como Prácticas de Gestión del Conocimiento de acuerdo con la literatura del área, que defina las Prácticas de Gestión del Conocimiento como destinadas a producir, retener, diseminar, compartir y aplicar el conocimiento en el ámbito de la organización y en la relación de ella con el ambiente en el cual se inserta.

c) La tercera parte del cuestionario, formada por una cuestión abierta, solicita al participante que relacione, de ser el caso, cuáles prácticas de la gestión del conocimiento merecen, en su visión, particular destaque por su importancia o frecuencia de utilización, relacionándolas a una o más prácticas entre las mencionadas en la segunda parte.

Las respuestas a estas cuestiones se basan en una escala social de Likert adaptada para capturar la percepción del participante con relación a los niveles de implantación y alcance de estas prácticas en el trabajo. De esta forma, para cada una de estas veinte cuestiones, el participante debe informar:

1. Su percepción sobre el estado de implantación de cada una de las veinte prácticas listadas. Para cada una de las respuestas se debe seleccionar apenas una de las siguientes opciones disponibles:

[0] - No existen planes de implantación

[1] - Planeadas para el futuro

[2] - Están en proceso de implantación

[3] - Ya fueron implantadas

[4] - Ya están implantadas y presentan resultados importantes y relevantes

2. Su percepción con relación al alcance de estas mismas prácticas en el ámbito de la organización. Para cada una de las respuestas se debe seleccionar apenas una de las siguientes opciones disponibles:

[0] - Sin planes para esta Prácticas de Gestión del Conocimiento

[1] - Apenas iniciativas aisladas

[2] - Restricta a apenas algunas secciones

[3] - En el ámbito de una división

[4] - Ampliamente diseminada en la organización

En la distribución del cuestionario a la población seleccionada, previamente fue enviado un mensaje estandarizado por email a cada participante seleccionado, solicitando su aceptación en participar de la investigación. A los que respondieron afirmativamente a la invitación, fue enviada, también por correo electrónico, una copia del cuestionario. El 06/02/2012 fueron enviados dieciséis cuestionarios a aquellos que aceptaron la invitación y, hasta el 09/03/2012, fueron recibidos diez cuestionarios correctamente respondidos, lo que resulta en $62,5 \%$ de respuestas positivas.

La Tabla 4 describe el perfil de los que respondieron el cuestionario, conteniendo la clase de empleado (civil o militar), el departamento en el cual actúa, el nivel de escolaridad y si ya recibieron algún tipo de entrenamiento formal en Gestión del Conocimiento. En la Tabla 4:

Clase de servidor: (M)ilitar o (C)ivil;

Departamento: Sólo los departamentos cuya actividad se ha convertido en relevante para a investigación;

Escolaridad: (S)uperior, (M)aestría y (D)octorado; y

Entrenamiento en Gestión del Conocimiento: (S)i o (N)o 
Tabla 4. Perfil de los participantes de la investigación

\begin{tabular}{|c|c|c|c|c|}
\hline No $^{\circ}$ & Clase & Departamento & Escolaridad & Entrenado en GC \\
\hline 1 & $\mathrm{M}$ & Coordinador Telemática & $\mathrm{M}$ & $\mathrm{N}$ \\
\hline 2 & $\mathrm{M}$ & Planificación de Asesoramiento y Gestión & $\mathrm{M}$ & $\mathrm{N}$ \\
\hline 3 & $\mathrm{M}$ & Coordinador de la Investigación y el Desarrollo & $\mathrm{S}$ & $\mathrm{N}$ \\
\hline 4 & $\mathrm{M}$ & Coordinador Telemática & $\mathrm{S}$ & $\mathrm{N}$ \\
\hline 5 & $\mathrm{M}$ & Coordinador Telemática & $\mathrm{S}$ & $\mathrm{N}$ \\
\hline 6 & $\mathrm{C}$ & Planificación de Asesoramiento y Gestión & $\mathrm{M}$ & $\mathrm{N}$ \\
\hline 7 & $\mathrm{C}$ & Coordinador Telemática & $\mathrm{S}$ & $\mathrm{N}$ \\
\hline 8 & $\mathrm{C}$ & Coordinador Telemática & $\mathrm{M}$ & $\mathrm{N}$ \\
\hline 9 & $\mathrm{C}$ & División de Sistemas & $\mathrm{M}$ & $\mathrm{S}$ \\
\hline 10 & $\mathrm{C}$ & División de Sistemas & $\mathrm{M}$ & $\mathrm{S}$ \\
\hline
\end{tabular}

\section{ANÁLISIS DE LOS RESULTADOS}

Para un mejor análisis de los resultados, se realizó un enfoque cuantitativo para establecer el Ranking Medio (RM) al cuestionario que utilizó la escala de Likert de 5 puntos para medir el grado de acuerdo de los sujetos que respondieron a los cuestionarios. Hemos llevado a cabo la verificación sobre el acuerdo o desacuerdo de las cuestiones evaluadas por la obtención de los resultados de RM concedidas a las respuestas relativas a la frecuencia de las respuestas de los encuestados que hicieron tal asignación, donde los valores inferiores a 3 se consideraron discordantes y más 3 que, como de común acuerdo, teniendo en cuenta una escala de 5 puntos. El valor exacto de 3 se considera "ya fueron implantadas" sobre el estado de implantación y en "el ámbito de una división" con relación al alcance, y el "neutral", lo que equivale a los casos en los encuestados dejan en blanco. Para el cálculo de la RM, utiliza el método de análisis de la escala de Likert presentado por Malhotra (2004)

La primera pregunta abierta de la investigación, ¿Qué entiende sobre Gestión del Conocimiento?, respondida por $50 \%$ de los participantes, mostró que apenas un (01) participante posee el correcto entendimiento con relación a lo que es la Gestión del Conocimiento. Este dato fue obtenido por simple comparación de las respuestas con la definición adoptada en la revisión bibliográfica.

La Tabla 5 presenta la precepción de los participantes con relación al estado de implantación de las Prácticas de Gestión del Conocimiento tratadas en la segunda parte del cuestionario y el Ranking Medio (RM). Se observa que el orden de presentación de las PGC en esta tabla es exactamente la misma del cuestionario. La Figura 1 presenta el gráfico radar para el estado de implantación.

En la tercera parte del cuestionario se propone una cuestión abierta con el siguiente texto: En el siguiente cuadro, cite las Prácticas de Gestión del Conocimiento relacionadas con una o más iniciativas de las mencionadas en el cuestionario y que, en su parecer, merecen destaque por su importancia o uso frecuente. Informe su nombre y una breve descripción de su objetivo. En el campo Prácticas de Gestión del Conocimiento indique el número de la práctica descrita en el cuestionario. Con esta pregunta se pretendía identificar y caracterizar las posibles prácticas de gestión del conocimiento formalizadas y de uso ampliamente diseminado y frecuente en el CTEx. Apenas un participante interpretó correctamente lo que se deseaba con esa cuestión y citó como Prácticas de Gestión del Conocimiento relevante un aplicativo de gerencia electrónica de documentos denominado SPED (Sistema de Protocolo Electrónico de Documentos), desarrollado en el CDS - Centro de Desarrollo de Software, órgano subordinado al Departamento de Ciencia y Tecnología (DCT).

La Tabla 6 presenta la percepción de los participantes con relación al alcance pretendido para las Prácticas de Gestión del Conocimiento tratadas en la segunda parte del cuestionario. Al igual que en la tabla anterior, el orden de presentación en esta tabla es exactamente la misma del cuestionario. La Figura 2 presenta el gráfico radar para el estado de alcance. 
Tabla 5. Respuestas obtenidas con relación al estado de implantación de las Prácticas de Gestión del Conocimiento tratadas

\begin{tabular}{|c|c|c|c|c|c|c|c|}
\hline \multirow{2}{*}{$\mathrm{N}^{\mathrm{O}}$} & \multirow{2}{*}{ Práctica } & \multicolumn{6}{|c|}{ Estado } \\
\hline & & 0 & 1 & 2 & 3 & 4 & RM \\
\hline 1 & Foros (presenciales y virtuales)/Listas de discusión & 6 & 1 & 1 & 1 & 1 & 1,00 \\
\hline 2 & Comunidades de Práctica/Comunidades de conocimiento & 6 & 2 & 0 & 2 & 0 & 0,80 \\
\hline 3 & Educación Corporativa & 6 & 1 & 3 & 0 & 0 & 0,70 \\
\hline 4 & Narrativas & 5 & 0 & 2 & 3 & 0 & 1,30 \\
\hline 5 & Mentoring & 7 & 1 & 1 & 0 & 1 & 0,70 \\
\hline 6 & Coaching & 7 & 1 & 1 & 0 & 1 & 0,70 \\
\hline 7 & Universidad Corporativa & 6 & 1 & 1 & 0 & 2 & 1,10 \\
\hline 8 & Mejores prácticas & 5 & 0 & 1 & 4 & 0 & 1,40 \\
\hline 9 & Benchmarking Interno y Externo & 6 & 0 & 3 & 1 & 0 & 0,90 \\
\hline 10 & Memoria Organizacional/Lecciones Aprendidas/Banco de Conocimientos & 4 & 2 & 1 & 2 & 1 & 1,40 \\
\hline 11 & Sistemas de Inteligencia Organizacional - Inteligencia Competitiva & 5 & 3 & 1 & 1 & 0 & 0,80 \\
\hline 12 & Mapeo o Auditoria del Conocimiento & 6 & 1 & 2 & 0 & 1 & 0.90 \\
\hline 13 & Sistema de Gestión por Competencias & 8 & 1 & 0 & 1 & 0 & 0,40 \\
\hline 14 & Banco de Competencias Organizacionales & 6 & 3 & 0 & 1 & 0 & 0,60 \\
\hline 15 & Banco de Competencias Individuales/Banco de Talentos/ Páginas Amarillas & 6 & 4 & 0 & 0 & 0 & 0,40 \\
\hline 16 & Gestión del Capital Intelectual/Gestión de los Activos Intangibles & 7 & 2 & 1 & 0 & 0 & 0,40 \\
\hline 17 & Herramientas de Colaboración como Portales, Intranets y Extranets & 1 & 1 & 1 & 4 & 3 & 2,70 \\
\hline 18 & Sistemas de Workflow & 4 & 1 & 2 & 2 & 1 & 1,50 \\
\hline 19 & Gestión de Contenido & 5 & 2 & 5 & 1 & 0 & 1,15 \\
\hline 20 & Gestión Electrónica de Documentos & 0 & 0 & 2 & 5 & 3 & 3,10 \\
\hline
\end{tabular}

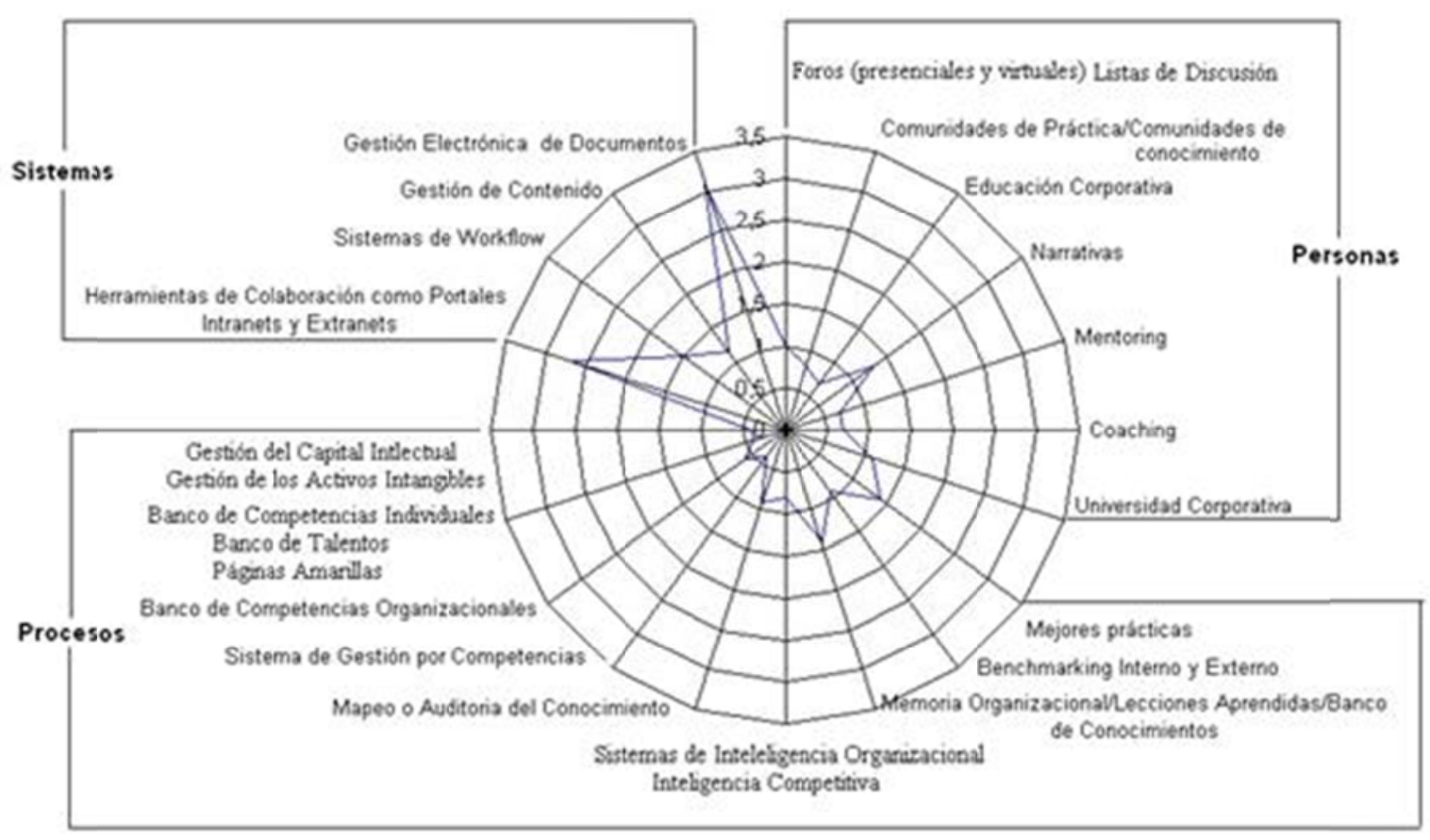

Fig. 1 : Respuestas el estado de implantación 
Tabla 6. Respuestas obtenidas con relación al alcance pretendido de las Prácticas de Gestión del Conocimiento tratadas

\begin{tabular}{|c|c|c|c|c|c|c|c|}
\hline \multirow{2}{*}{$\mathrm{N}^{\circ}$} & \multirow{2}{*}{ Práctica } & \multicolumn{6}{|c|}{ Alcance } \\
\hline & & 0 & 1 & 2 & 3 & 4 & RM \\
\hline 1 & Foros (presenciales y virtuales)/Listas de discusión & 5 & 1 & 0 & 2 & 2 & 1,50 \\
\hline 2 & Comunidades de Práctica/Comunidades de conocimiento & 2 & 6 & 0 & 0 & 2 & 1,40 \\
\hline 3 & Educación Corporativa & 3 & 0 & 4 & 1 & 2 & 1,90 \\
\hline 4 & Narrativas & 2 & 2 & 1 & 1 & 4 & 2,30 \\
\hline 5 & Mentoring & 4 & 4 & 0 & 1 & 1 & 1,10 \\
\hline 6 & Coaching & 5 & 2 & 2 & 0 & 1 & 1,00 \\
\hline 7 & Universidad Corporativa & 5 & 2 & 0 & 0 & 3 & 1,40 \\
\hline 8 & Mejores Prácticas & 2 & 4 & 2 & 1 & 1 & 1,50 \\
\hline 9 & Benchmarking Interno y Externo & 3 & 5 & 0 & 0 & 2 & 1,30 \\
\hline 10 & Memoria Organizacional/Lecciones Aprendidas/Banco de Conocimientos & 1 & 2 & 2 & 1 & 4 & 2,50 \\
\hline 11 & Sistemas de Inteligencia Organizacional - Inteligencia Competitiva & 4 & 2 & 2 & 1 & 1 & 1,30 \\
\hline 12 & Mapeo o Auditoria del Conocimiento & 5 & 1 & 2 & 1 & 1 & 1,20 \\
\hline 13 & Sistema de Gestión por Competencias & 7 & 1 & 0 & 1 & 1 & 0,80 \\
\hline 14 & Banco de Competencias Organizacionales & 5 & 1 & 2 & 0 & 2 & 1,30 \\
\hline 15 & Banco de Competencias Individuales/Banco de Talentos/ Páginas Amarillas & 5 & 1 & 1 & 0 & 3 & 1,50 \\
\hline 16 & Gestión del Capital Intelectual/Gestión de los Activos Intangibles & 6 & 1 & 3 & 0 & 0 & 0,70 \\
\hline 17 & Herramientas de Colaboración como Portales, Intranets y Extranets & 1 & 2 & 0 & 1 & 6 & 2,90 \\
\hline 18 & Sistemas de Workflow & 3 & 1 & 3 & 0 & 3 & 1,90 \\
\hline 19 & Gestión de Contenido & 5 & 1 & 2 & 1 & 1 & 1,20 \\
\hline 20 & Gestión Electrónica de Documentos & 0 & 1 & 0 & 2 & 7 & 3,50 \\
\hline
\end{tabular}

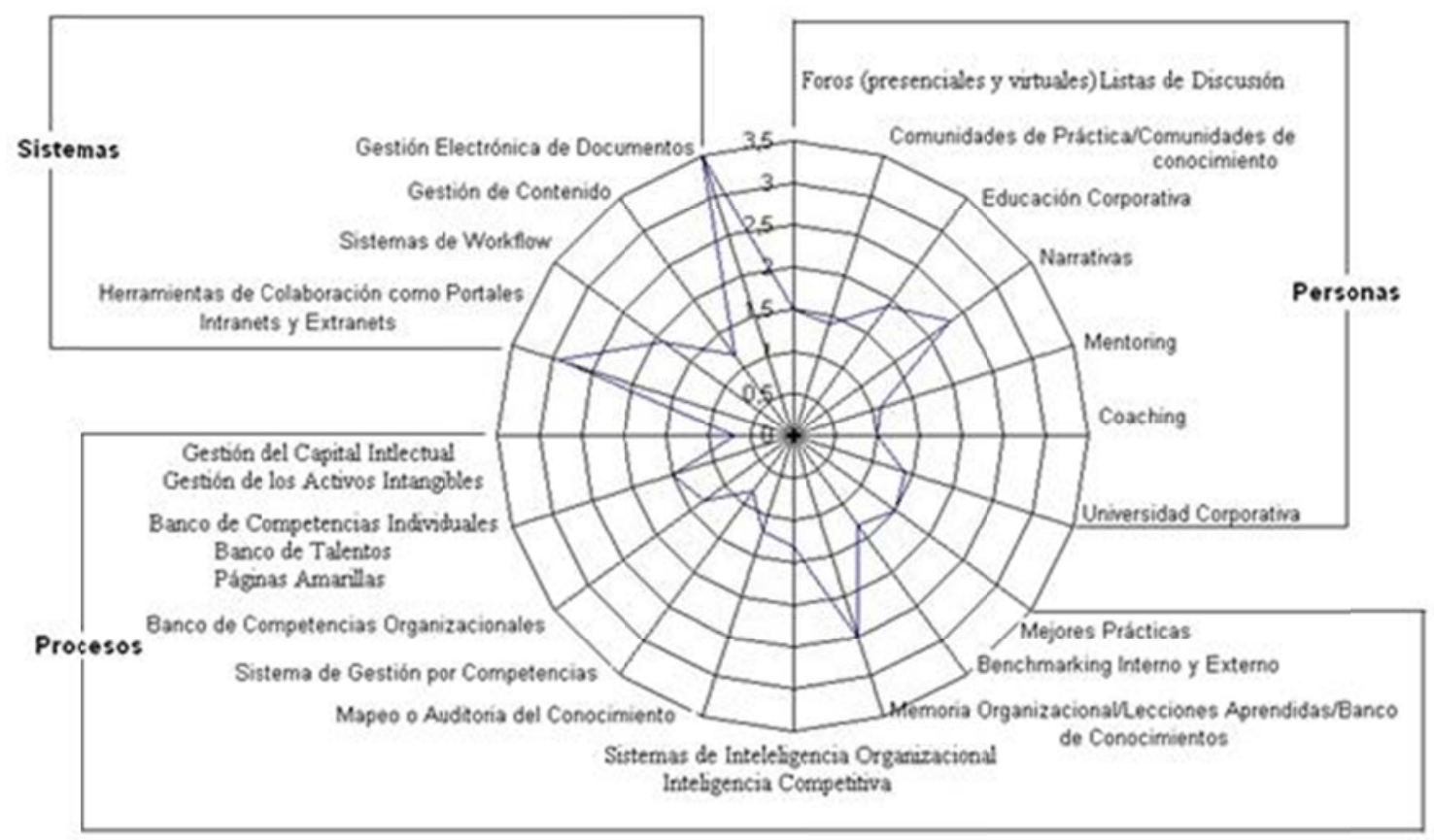

Fig. 2: Respuestas el estado de alcance

En la categoría Personas, destaca la Prácticas de Gestión del Conocimiento "Narrativas" como la práctica con el más alto nivel de implementación y alcance. En la categoría Procesos, destacamos las Prácticas de Gestión del Conocimiento "Mejores prácticas" y "Memoria Organizacional/Lecciones Aprendidas/Banco de Conocimientos" como las prácticas con niveles más altos de implementación y alcance. Las prácticas "Banco de Competencias Individuales/Banco de Talentos/ Páginas Amarillas" y "Gestión del Capital Intelectual/Gestión de los Activos Intangibles" no se obtiene ningún relato de efectiva implantación. 
En la categoría Sistemas, destacamos las Prácticas de Gestión del Conocimiento "Gestión Electrónica de Documentos" y "Herramientas de Colaboración como Portales, Intranets y Extranets" como las prácticas con niveles más altos de implementación y alcance. Con relación a la tercera parte del cuestionario de recolección de datos para la investigación, tan importante como relatar el número irrisorio de respuestas coherentes recibidas (apenas un participante respondió correctamente la pregunta) es concluir que 90\% de los entrevistados no poseen ninguna idea sobre lo que representa la Gestión del Conocimiento para la institución.

\section{CONCLUSIONES}

Después de examinar los resultados obtenidos, se verificó la no existencia de un Planeamiento Formal con relación a la Gestión del Conocimiento. Por ser la institución evaluada una organización militar, podemos también afirmar que este resultado se extiende a todos sus departamentos, ya que el modelo organizacional es centralizado y determinado por los escalones superiores, no habiendo margen para que esto sea diferente.

Entre los veinte prácticas de gestión del conocimiento estudiados puede concluir que sólo Gestión Electrónica de Documentos y Herramientas de Colaboración como Portales, Intranet y Extranet son de implementación de alto nivel.

Del mismo modo, entre los veinte estudiadas, sólo las prácticas de Mejores Prácticas y Memoria Organizacional / Lecciones Aprendidas / Banco de Conocimientos están en el nivel medio de la implementación.

Tres prácticas se destacan de los demás porque no han recibido ningún informe de aplicación efectiva a saber: Educación Corporativa, Banco de Competencias Individuales / Banco de Talentos / Páginas Amarillas y Gestión del Capital Intelectual / Gestión de los Activos intangibles..

\section{REFERENCIAS}

Alee, V. y Taug, J., Collaboration, innovation and value creation in a global telecom. The Learning Organization, v.13, n.6, (2006).

Amidon, D. M.R. The Challenge of $5^{\text {th }}$ Generation R\&D, Research Technology Management. Journal of the Industrial Research Institute, Jul-Ago, (1996).

Ansuattigui, R.V.; Pithon, A.J.C., Aplicação da Metodologia OKA em Redes Colaborativas de Autoria. XXXI Encontro Nacional de Engenharia de Produção (ENEGEP), 1-12, Belo Horizonte, Brasil, 4 al 7 de octubre (2011).

Ansuattigui, R. V. Estudo das Práticas de Gestão do Conhecimento em uma Instituição Pública de Pesquisa: O caso do Centro Tecnológico do Exército. Dissertação de Mestrado, Mestrado em Tecnologia, Centro Federal de Educação Tecnológica, Rio de Janeiro, RJ, Brasil, (2012).

Batista, F. F.; Quandt, C. O.; Pacheco, F. F.; Terra, J. C. C. Gestão do Conhecimento na Administração Pública. Texto para Discussão no 1095. Brasília: IPEA, (2005).

Burnett, Simon; Illingworth, Lorraine; Webster, Linda. Knowledge Auditing and Mapping: A Pragmatic Approach. Knowledge and Process Management, v.11, n.1, pp.25-37, (2004).

Camões, R. J. S. Proposta e Teste de um Método de Aplicação do Método Organizational Knowledge Assessment - OKA: Estudo de Caso em Organização Militar. Dissertação de Mestrado, Programa de PósGraduação em Gestão do Conhecimento e da Tecnologia da Informação, Universidade Católica de Brasília, Brasília, DF, Brasil, (2010).

Cheung, C.F. et. al. A Systematic Approach for Knowledge Auditing: A Case Study in Transportation Sector. Journal of Knowledge Management, v.11, n.4, pp. 140-158, (2007).

Coombs, R.; Hull, R.; Peltu, M. Knowledge Management Practices for Innovation: An audit tool for improvement.CRIC Working Paper $N^{\circ} 6$. Centre for Research on Innovation and Competition, The University of Manchester. June, (1998). 
Davenport, T. H. y Prusak, L., Conhecimento Empresarial: como as organizações gerenciam o seu capital intellectual. Rio de Janeiro: Elsevier, (2003).

Henczel, S., The Information Audit As A First Step Towards Effective Knowledge Management: An Opportunity for The Special Librarian. Inspel, v.34, pp. 210-226, (2000).

Hylton, A., A KM Initiative is Unlikely to Succeed without a Knowledge Audit, 2002. http://www.providersedge.com/docs/km_articles/km_initiative_unlikely_to_succeed_without_a_k_audit.pdf. Acceso: 10 septiembre (2011).

Liebowitz, Jaw et. al. The Knowledge Audit Knowledge and Process Management, v.7,n.1, pp. 3-10, (2000). Malhotra, N. Marketing Research: An Applied Orientation. Prentice Hall, (2004).

Monroy, Martín E; Arciniegas, José L y Rodriguez, Julio C. Caracterización de Herramientas de Ingeniería Inversa. Inf. tecnol. [online]. 2012, vol.23, n.6 pp. 31-42. Disponível em: <http://www.scielo.cl/scielo.php?script=sci_arttext\&pid=S0718-07642012000600005\&lng=pt\&nrm=iso>. ISSN 0718-0764. doi: 10.4067/S0718-076̄2012000600005.

Neves, W. Gestão do Conhecimento na Administração Pública Federal: Estudo de Caso no Centro Tecnológico do Exército. Dissertação de Mestrado, Mestrado em Administração e Desenvolvimento Empresarial da Universidade Estácio de Sá, Rio de Janeiro, RJ, Brasil, (2011).

Nonaka, I., A Dynamic Theory of Organizational Knowledge Creation. Organization Science, v.5, n.1, February (1994).

Park, Y.; Kim, S. Linkage between knowledge management and R\&D management. Journal of Knowledge Management, v.9, n.4, (2005).

Perez-Soltero, A. et. al. "Knowledge Audit Methodology with Emphasis on Core Processes". European and Mediterranean Conference on Information Systems (EMCIS), Costa Blanca, Alicante, Spain, 6-7 julio (2009).

Quigley, E. J., \& Debons, A. Interrogative theory of information and knowledge. Proceeding of the 1999 ACM SIGCPR conference on Computer personal research, New Orleans, Lousiana, United States, (1999).

Stenmark, D., The Relationship between Information and Knowledge. Proceedings of IRIS 24, Ulvik, Norway, August 11-14 (2001).

Suarez, Víctor F; Guerrero, Álvaro y Castrillon, Omar D. Programación de Horarios Escolares basados en Ritmos Cognitivos usando un Algoritmo Genético de Clasificación No-dominada, NSGA-II. Inf. tecnol. [online]. 2013, vol.24, n.1, pp. 103-114. Disponível em: <http://www.scielo.cl/scielo.php?script=sci_arttext\&pid=S0718-07642013000100012\&lng=pt\&nrm=iso>. ISSN 0718-0764. doi:10.4067/S0718-07642013000100012.

Sveiby, K.E., Knowledge Management - Lessons from the pioneers. San Francisco, 2001. http://www.providersedge.com/docs/km_articles/KM_-_Lessons_from_the_Pioneers.pdf. Acesso: 19 noviembre (2011).

Tigre, P. B., Gestão da Inovação - A Economia da Tecnologia no Brasil. Rio de Janeiro: Campus, (2006).

Toffler, A., A Terceira onda. Rio de Janeiro, Record, Brasil (1980).

Wiig, K. M., Knowledge Management: Na Emerging Discipline Rooted in a Long History: Knowledge Research Institute, Inc, (1999). 